\title{
3D Thermography for the Measurement of Surface Heat Dissipation
}

\author{
Robert Schmoll1, Sebastian Schramm ${ }^{1}$, Tom Breitenstein ${ }^{1}$, Andreas Kroll ${ }^{1}$ \\ ${ }^{1}$ University of Kassel, Department of Measurement and Control, \\ Mönchebergstraße 7, 34109 Kassel, Germany, \\ robert.schmoll@mrt.uni-kassel.de
}

\begin{abstract}
Summary:
3D thermal imaging can be realized by sensor data fusion of a depth and a thermal camera. In the resulting 3D thermogram thermal and spatial information of an object is available. This information is used to calculate the surface heat dissipation caused by thermal radiation and natural convection.
\end{abstract}

Keywords: 3D Thermography, Sensor Data Fusion, Heat Dissipation, Heat Loss

\section{Introduction}

In contrast to the identification of thermal bridges and insulation leaks by qualitative thermal imaging it is hard to determine quantitative heat flows through object surfaces. If the geometry and the surface temperature of an object are known, it is possible to calculate the heat dissipation (or the heat loss) by thermal radiation and natural convection. A 3D thermography system enables the simultaneous measurement of the required information.

3D thermography systems differ mainly by the acquisition of the geometric 3D data. Especially for outdoor scenes, e.g. to scan buildings, laser scanners are used [1]. For reasons of scanning speed and costs, it is possible to use so called depth cameras [2]. These cameras work with structured light, often in the near-infrared (NIR) range. They have a limited range (approx. up to $10 \mathrm{~m}$ ) and, caused by the sensitivity for external NIR radiation, are mainly used for indoor measurements [2].

\section{D Thermography System}

The used 3D thermography system in this work consists of a long-wave infrared (LWIR) camera and a depth camera, see Fig. 1 and [4] for details. The thermal information of the LWIR camera has to be projected into the geometric 3D data (e.g. point clouds) of the depth camera. To avoid holes in the 3D data caused by occlusion at one viewing angle it is beneficial to capture the object from different views, e.g. by moving the measurement system around the object. For this reason, multiple point clouds resulting from different viewing angles have to be registered into a single 3D model.

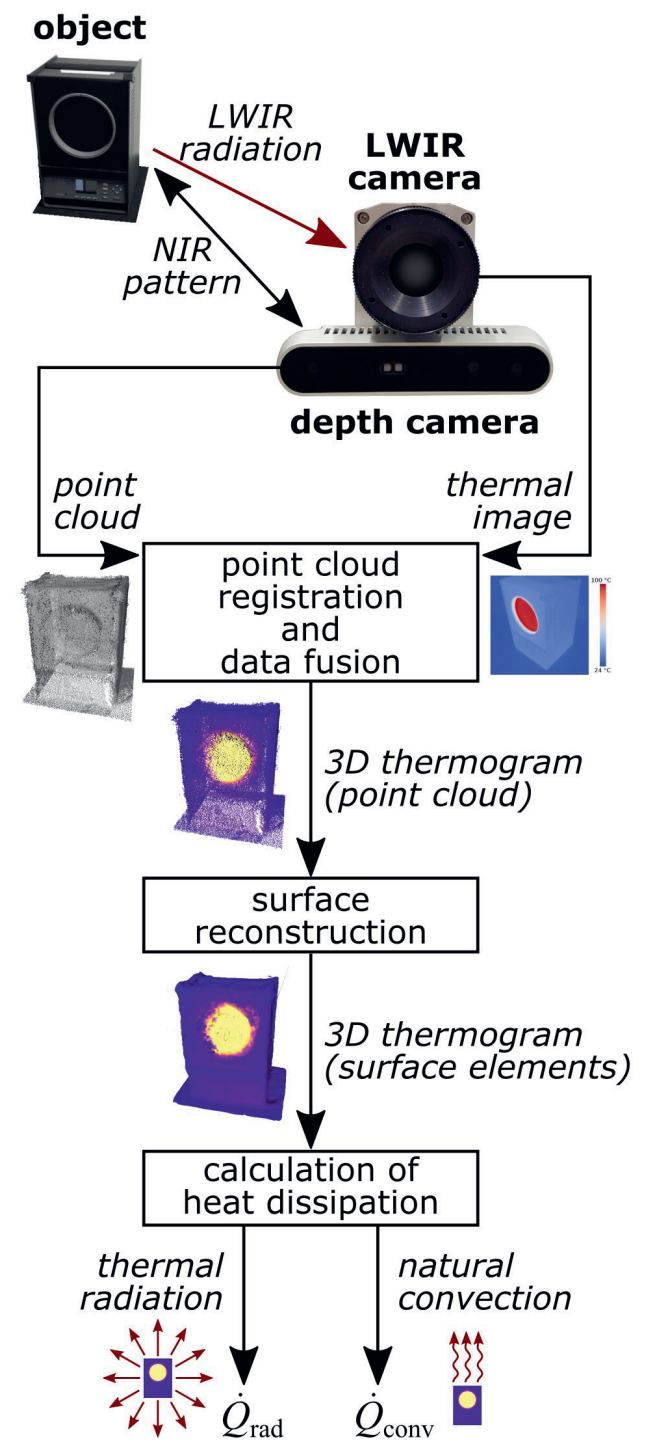

Fig. 1. Workflow of measuring surface heat dissipation using $3 D$ thermography. 
The point cloud registration and the fusion of spatial and thermal information is carried out in real-time. Therefore, self-localization and mapping algorithms, running on a graphics card of a portable high-performance computer, have to be adapted to include the thermal data [3]. For this task, the intrinsic and extrinsic calibration of the involved cameras is needed [4].

In the next step, the point cloud is converted to surface elements by a Poisson surface reconstruction [5]. For these surface elements, it is possible to calculate the radiated heat power and the heat transport by natural convection.

\section{Calculation of Heat Dissipation}

After this step, the 3D thermogram consists of surface elements (triangles) with the temperature $T_{i}, i=1 \ldots N$. For the temperature measurement, the object was assumed as a grey body and the emissivity $\varepsilon_{i}$ of each surface has to be determined. The area $A_{i}$ of each surface element is known from the geometric data. At least, the Stefan-Boltzmann constant $\sigma$ and the ambient temperature $T_{\text {amb }}$ have to be known to calculate the heat flow caused by thermal radiation [6]:

$$
\dot{Q}_{\mathrm{rad}}=\sum_{i=1}^{N} A_{i} \cdot \varepsilon_{i} \cdot \sigma \cdot\left(T_{i}^{4}-T_{\mathrm{amb}}^{4}\right)
$$

In order to calculate the heat flow by natural convection the air temperature $T_{\text {air }}$ is needed. For the determination of the heat transfer coefficient $\alpha_{i}$ each surface element $i$ is treated separately and assumed to be alone in an infinite space with known orientation to the gravity. With this information the heat flow by natural convection is calculated by [6]:

$$
\dot{Q}_{\text {conv }}=\sum_{i=1}^{N} A_{i} \cdot \alpha_{i} \cdot\left(T_{i}-T_{\text {air }}\right)
$$

\section{Test Object for Verification}

An infrared radiator is chosen as a test object, see Fig. 2. The emissivities $\varepsilon_{\text {front }}=0.95$ and $\varepsilon_{\text {rear }}=0.84$ were determined by the use of reference tape.

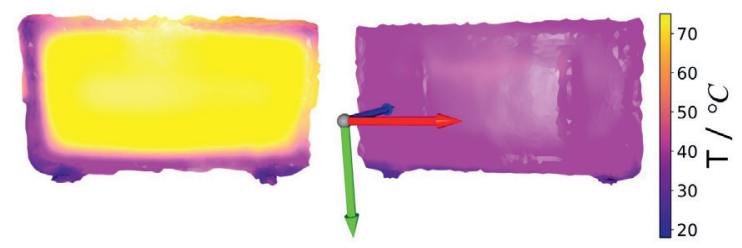

Fig. 2. Front (left) and rear (right) view of a $3 D$ thermogram (surface elements) of an infrared radiator.
The results of the measured heat dissipation could be compared to the electrical power consumption measured by a wattmeter, see Tab. 1 .

Tab. 1: Calculated heat dissipation of the infrared radiator and power consumption.

\begin{tabular}{|c|c|c|}
\hline & Measured & Reference \\
\hline $\begin{array}{c}\text { Surface } \\
\text { area }\end{array}$ & $1.66 \mathrm{~m}^{2}$ & $\sim 1.60 \mathrm{~m}^{2}$ \\
\hline $\begin{array}{c}\text { Heat dis- } \\
\text { sipation }\end{array}$ & $618.1 \mathrm{~W}$ & $612.0 \mathrm{~W}$ \\
\hline
\end{tabular}

\section{Conclusions}

The used test object gives very good results for the measurement of the surface area and the heat dissipation. An uncertainty analysis has not been carried out.

\section{Summary and Outlook}

In this work the information of a 3D thermogram is used, to calculate the heat dissipation of scanned objects. It should be mentioned, that internal heat flows, forced convection or the use of e.g. slits in the surface could not be covered by the measuring system. Additionally, newer algorithms of point cloud registration, see [3], allow 3D thermograms of larger objects. Finally, the method enables the direct measurement of surface heat dissipation by thermal radiation and natural convection e.g. to calculate losses and to optimize the efficiency.

\section{References}

[1] A. Costanzo, M. Minasi, G. Casula, M. Musacchio, M. F. Buongiorno, Combined use of terrestrial laser scanning and IR thermography applied to a historical building, MDPI Sensors, 15(1), 194-213 (2015); doi: 10.3390/s150100194

[2] A. O. Ordonez Müller, A. Kroll, Generating high fidelity 3-D thermograms with a handheld realtime thermal imaging system, IEEE Sensors Journal, 17(3), 774-783 (2016); doi: 10.1109/JSEN.2016.2621166

[3] S. Schramm, P. Osterhold, R. Schmoll, A. Kroll, Generation of Large-Scale 3D Thermograms in Real-Time Using Depth and Infrared Cameras, In 15th Quantitative InfraRed Thermography Conference (QIRT), Porto, Portugal, 6.-10. July (2020)

[4] S. Schramm, J. Rangel, D. A. Salazar, R. Schmoll, A. Kroll, Target Analysis for the Multispectral Geometric Calibration of Cameras in Visual and Infrared Spectral Range, IEEE Sensors Journal, to appear in print (2020); doi: 10.1109/JSEN.2020.3019959

[5] M. Kazhdan, H. Hoppe, Screened poisson surface reconstruction, ACM Transactions on Graphics, 32, 1-13 (2013); doi: 10.1145/2487228.2487237

[6] VDI-Wärmeatlas, 11. Auflage, Springer Berlin Heidelberg (2013) 